\title{
Reduced Grey Matter Volume in Frontal and Temporal Areas in Depression: A Voxel Based Morphometry Study
}

\author{
Sevdalina Kandilarova ${ }^{1,2}$, Drozdstoy Stoyanov ${ }^{1,2}$, Nickolay Sirakov ${ }^{1,3}$, Michael Maes ${ }^{2}$, \\ Karsten Specht ${ }^{4,5}$ \\ ${ }^{1}$ Research Complex for Translational Neuroscience, Medical University of Plovdiv (MUP), \\ Plovdiv, Bulgaria \\ ${ }^{2}$ Department of Psychiatry and Medical Psychology, MUP \\ ${ }^{3}$ Department of Image Diagnostics, Dental Allergology and Physiotherapy, MUP \\ ${ }^{4}$ Department of Biological and Medical Psychology, University of Bergen, Bergen, Norway \\ ${ }^{5}$ Department of Education, UiT/The Arctic University of Norway, Tromsø, Norway
}

Running title: Reduced grey matter volume in depression

Sevdalina Kandilarova: kandilarova@gmail.com

Drozdstoy Stoyanov: stojanovpisevski@gmail.com

Nickolay Sirakov: nsirakov@gmail.com

Michael Maes: dr.michaelmaes@hotmail.com

Karsten Specht: Karsten.Specht@uib.no

\begin{abstract}
Objective: The aim of the current study was to examine whether and to what extent mood disorders, comprising major depression and bipolar disorder, are accompanied by structural changes in the brain as measured using voxel-based morphometry (VBM). Methods: We have performed a VBM study using a 3T MRI system (GE Discovery 750w) in patients with mood disorders $(\mathrm{n}=50)$, namely 39 with major depression and 11 with bipolar disorder, compared to 42 age, sex and education matched healthy controls. Results: Our results show that depression was associated with significant decreases in grey matter (GM) volume restricted to regions located in medial frontal and anterior cingulate cortex on the left side and middle frontal gyrus, medial orbital gyrus, inferior frontal gyrus (triangular and orbital parts), and middle temporal gyrus (extending to the superior temporal gyrus) on the right side. When the patient group was separated into bipolar disorder and major depression the reductions remained significant only for the patients with major depressive disorder. Conclusions: Using VBM the present study was able to replicate decreases in GM volume restricted to frontal and temporal regions in patients with mood disorders mainly major depression, as compared with healthy controls.
\end{abstract}

Keywords: major depressive disorder, bipolar disorder, structural MRI, grey matter volume, voxel-based morphometry 
Significant outcomes

1. Unipolar major depression is characterized by decrease in anterior cingulate cortex grey matter explaining in part aberrations in cognitive and affective regulation, attention, problem solving, motivation, and decision making.

2. Unipolar major depression is characterized by decreases in orbitofrontal cortex grey matter explaining aberrations in social and emotional behaviours including processing of rewards and punishment.

Limitations

1. The bipolar disorder study sample was relatively small.

2. Recently, major biological differences were detected between BD1 and BD2 patients, and therefore, future MRI research should examine differences among both BD subtypes.

3. Enhanced neuro-oxidative pathways with neurodegenerative effects are more expressed in unipolar depressed that in bipolar patients and therefore future research should examine decreased grey matter volume in mood disorders in association with neuro-oxidative pathways.

\section{Introduction}

Depression is one of the most common psychiatric disorders with increasing frequency, and immense social, and economic burden ${ }^{1}$. The clinical representation encompasses affective, cognitive and somatic complains such as low mood, anxiety, lack of pleasure and interest, disturbances of sleep and appetite, decreased self-esteem, thoughts of guilt, hopelessness, and even suicide. Important insight into the underlying pathophysiological mechanisms has been gained through recent advances in both structural and functional neuroimaging but many issues remain to be resolved ${ }^{2,3}$.

One major such problem is the question of whether and to what extent depression causes structural changes in the brain. The majority of the individual studies and meta-analyses published in this field are positive about gray matter (GM) volume reductions in various cortical regions in depressed patients (unipolar and bipolar alongside) compared to healthy controls ${ }^{4-7}$. The most consistent findings appear to be related to GM loss in medial frontal cortex (MFC), anterior cingulate cortex (ACC) and orbitofrontal cortex (OFC) with less evidence about subcortical structures such as the amygdala and the hippocampus 4,8-11. Nevertheless, a number of studies have failed to detect some regional changes in GM volume (for instance ACC) while confirming others (OFC) ${ }^{12,13}$.

In addition, the exact localization (bilateral, left or right) and the extent of the GM reductions reported often varies from one study to another. For instance, the meta-analysis by Lai 6 detected GM loss in bilateral ACC, while Arnone et al. ${ }^{5}$ demonstrated a decrease only in right $\mathrm{ACC}$ in major depression. Bipolar depression, on the other hand, appears to affect the left ACC ${ }^{7}$. Similar controversies exist about the reduction of the GM in OFC spanning from bilateral engagement of its medial and lateral parts to only left or right sided loss ${ }^{4,5,14}$.

These and other inconsistencies can be explained by the heterogeneity of the patient samples (unipolar vs bipolar depression, severity, psychotic features, remission, medication status), variability of the image acquisition parameters, and flexibility of the methods employed (a priori defined regions of interest (ROIs) or whole brain analysis such as voxel-based morphometry). In an attempt to meet the crucial need for replication studies in the field of psychiatric neuroimaging we have performed a voxel-based morphometry study on a sample of depressed patients (both unipolar and bipolar) compared to matched group of healthy controls. 
We have assumed that in many cases the "unipolar" depression as defined in major depressive disorder (MDD) might be interpreted within the context of the bipolar spectrum as well ${ }^{15-17}$, especially when the hypomanic episodes have manifested on sub-threshold, latent or mixed syndrome level ${ }^{17-20}$ and therefore might have not been properly registered in medical records. Despite recent efforts to understand the underlying pathophysiological mechanisms the question of whether and to what extent mood disorders cause structural changes in the brain remains unclear.

Hence, the current study was carried out to examine a voxel-based morphometry (VBM) study on a sample of mood disorder patients comprising depression and bipolar disorder compared to matched group of healthy controls. Our initial hypothesis based on the findings of previous VBM studies was that we would find GM volume decreases in cortical areas related to emotion processing and cognitive functioning such as ACC, MFC, and OFC. Based on this assumption we first pooled the two patient groups together and compared them to the control subjects and subsequently we performed a separate one-way ANOVA analysis with the tree subsamples (unipolar, bipolar and healthy) in order to define the shared and distinct GM volume changes in patients suffering from those two major psychiatric disorders.

\section{Methods}

\section{Subjects}

For the present study we recruited fifty adult subjects (mean age $46.7 \pm 13.1,12$ males) complying with the DSM-IV-TR criteria for depressive episode (single or recurrent) of major depressive disorder $(n=39)$ or bipolar disorder $(n=11)$ and forty-two age and sex matched healthy controls (mean age $42.6 \pm 13.7 \mathrm{y}, 13$ males). All participants were assessed by general clinical interview and the structured Mini International Neuropsychiatric Interview (M.I.N.I 6.0) ${ }^{21}$. Montgomery-Åsberg Depression Rating Scale (MADRS) ${ }^{22}$ was used for assessment of severity. Subjects were excluded if they had a second axis-I diagnosis (psychotic, anxiety, substance related disorder) or severe suicidal risk (10th item of MADRS $\geq 2$ ).

Healthy controls did not comply with any of the DSM-IV-TR diagnoses included in the M.I.N.I., and had no history of any psychiatric disorder. Exclusion criteria for both patients and healthy controls were the following: major medical illness, neurological disease, history of head trauma with loss of consciousness. All participants provided a written informed consent complying with the Declaration of Helsinki and the study was approved by the University's Ethics Committee (P-1369/29.05.2015).

\section{MRI acquisition and analysis}

High resolution structural scans were obtained from all participants on a 3T MRI system (GE Discovery $750 \mathrm{w}$ ) with the following specifications - Sag 3D T1 FSPGR series, slice thickness $1 \mathrm{~mm}$, matrix $256 \times 256$, relaxation time (TR) - $7.2 \mathrm{msec}$, echo time (TE) - $2.3 \mathrm{msec}$, flip angle $12^{\circ}$.

MRI data were analysed using the SPM 12 (Statistical Paramertic Mapping, http://www.fil.ion.ucl.ac.uk/spm/) software running on MATLAB R2017 for Windows and the CAT 12 toolbox implemented in SPM (http://www.neuro.uni-jena.de/software/). The preprocessing included the following steps: i) segmentation through the CAT 12 toolbox, including normalization to standardized MNI (Montreal Neurological Institute) space, followed by ii) spatial smoothing with a $8 \mathrm{~mm}$ full-width-at-half-maximum Gaussian kernel. Total intracranial volume was calculated as well. For the statistical analysis, two generallinear models were defined. First, we compared the grey-matter volume between the two groups (Patients and Controls) with a two-sample t-test. Next, an ANOVA model was specified that further differentiated between subjects with MDD, subjects with bipolar 
disorder, and healthy control subjects. All statistical models included the total intracranial volume as covariate, and all results were explored with an FDR-corrected threshold of $\mathrm{p}<0.05$ (false discovery rate) and an extent threshold of at least 36 voxels per cluster (expected voxels per cluster).

\section{Statistical analysis}

We performed statistical analysis of the demographic and clinical characteristics of the participants using SPSS 22.0 for Windows. The level of significance was set to $p<0.05$ for all tests. Student's t-test was applied for continuous variables and Chi-square test - for categorical ones.

\section{Results}

\section{Demographic and clinical characteristics}

There were no statistically significant differences in age, sex and education level between the patients and the healthy controls. Expectedly, patients had significantly higher MADRS scores. Clinical characteristics of the patient sample are given in detail in Table 1.

Table 1. Demographic and clinical characteristics of the participants

\begin{tabular}{|c|c|}
\hline $\begin{array}{l}\text { Healthy controls } \\
(\mathrm{n}=42)\end{array}$ & $\begin{array}{l}\text { Patients } \\
(\mathrm{n}=50)\end{array}$ \\
\hline
\end{tabular}

\begin{tabular}{llll}
\hline Age $($ mean \pm SD) & $42.6 \pm 13.7$ & $46.4 \pm 13.9$ & $0.145^{\mathrm{a}}$ \\
Sex $(\mathrm{M} / \mathrm{F})$ & $13 / 29$ & $12 / 38$ & $0.488^{\mathrm{b}}$ \\
Education (secondary/higher) & $17 / 25$ & $25 / 25$ & $0.230^{\mathrm{b}}$ \\
MADRS score (mean $\pm \mathrm{SD})$ & $0.8 \pm 1.5$ & & $* 0.000^{\mathrm{a}}$
\end{tabular}

MDD/BD

$39 / 11$

Age at onset (years)

$34.9 \pm 12.3$

Number of previous episodes

$3.4 \pm 3.5$

Duration of illness (months)

$144.2 \pm 117.7$

SSRIs (N)

15

SSNRIs (N)

18

SNRIs (N) 
TCAs (N)

Benzodiazepines $(\mathrm{N})$

SD - Standard Deviation, ${ }^{\mathrm{a}}$ Independent samples t-test, ${ }^{\mathrm{b}} \chi^{2}$ - test, MADRS - Montgomery- $\AA$ sberg Depression Rating Scale, * $\mathrm{p}<0.05, \mathrm{~N}$ - number of patients, MDD - major depressive disorder, $\mathrm{BD}-$ bipolar disorder, SSRIs - selective serotonin reuptake inhibitors, SNRIs - selective noradrenalin inhibitors, SSNRI - selective serotonin-noradrenalin reuptake inhibitors, TCAs - tricyclic antidepressants.

\section{Voxel based morphometry (VBM)}

First, we performed a two-sample t-test on the grey-matter images with the total intracranial volume as covariate. The contrast between controls and patients yielded significant differences in clusters encompassing left medial frontal (MFC) and anterior cingulate cortex $(\mathrm{ACC})$ as well as right frontal and temporal regions $(\mathrm{p}(\mathrm{FDR})<0.05)$. The results are described in detail in Table 2 and an illustration is given in Figure 1. The reverse contrast exploration did not produce any surviving significant clusters.

Table 2. Clusters of grey matter volumes significantly reduced in all patients compared to healthy controls

\begin{tabular}{|c|c|c|c|c|c|}
\hline \multirow[t]{2}{*}{ Anatomical localization } & \multirow{2}{*}{$\begin{array}{l}\text { Cluster size } \\
\text { (voxels) }\end{array}$} & \multicolumn{3}{|c|}{ Peak MNI coordinates } & \multirow{2}{*}{$\begin{array}{l}\text { p-value } \\
\text { (FDR- } \\
\text { corrected) }\end{array}$} \\
\hline & & $\mathrm{x}$ & $\mathrm{y}$ & $\mathrm{z}$ & \\
\hline Left medial frontal cortex and & 435 & -12 & 56 & -6 & 0.018 \\
\hline anterior cingulate & & -15 & 44 & 9 & 0.018 \\
\hline Right middle frontal gyrus & 82 & 38 & 47 & -5 & 0.018 \\
\hline Right medial orbital gyrus & 70 & 14 & 51 & -12 & 0.025 \\
\hline $\begin{array}{l}\text { Right inferior frontal gyrus } \\
\text { (triangular and orbital parts) }\end{array}$ & 62 & 45 & 35 & 0 & 0.021 \\
\hline Right middle temporal gyrus & 56 & 51 & -6 & -22 & 0.018 \\
\hline
\end{tabular}




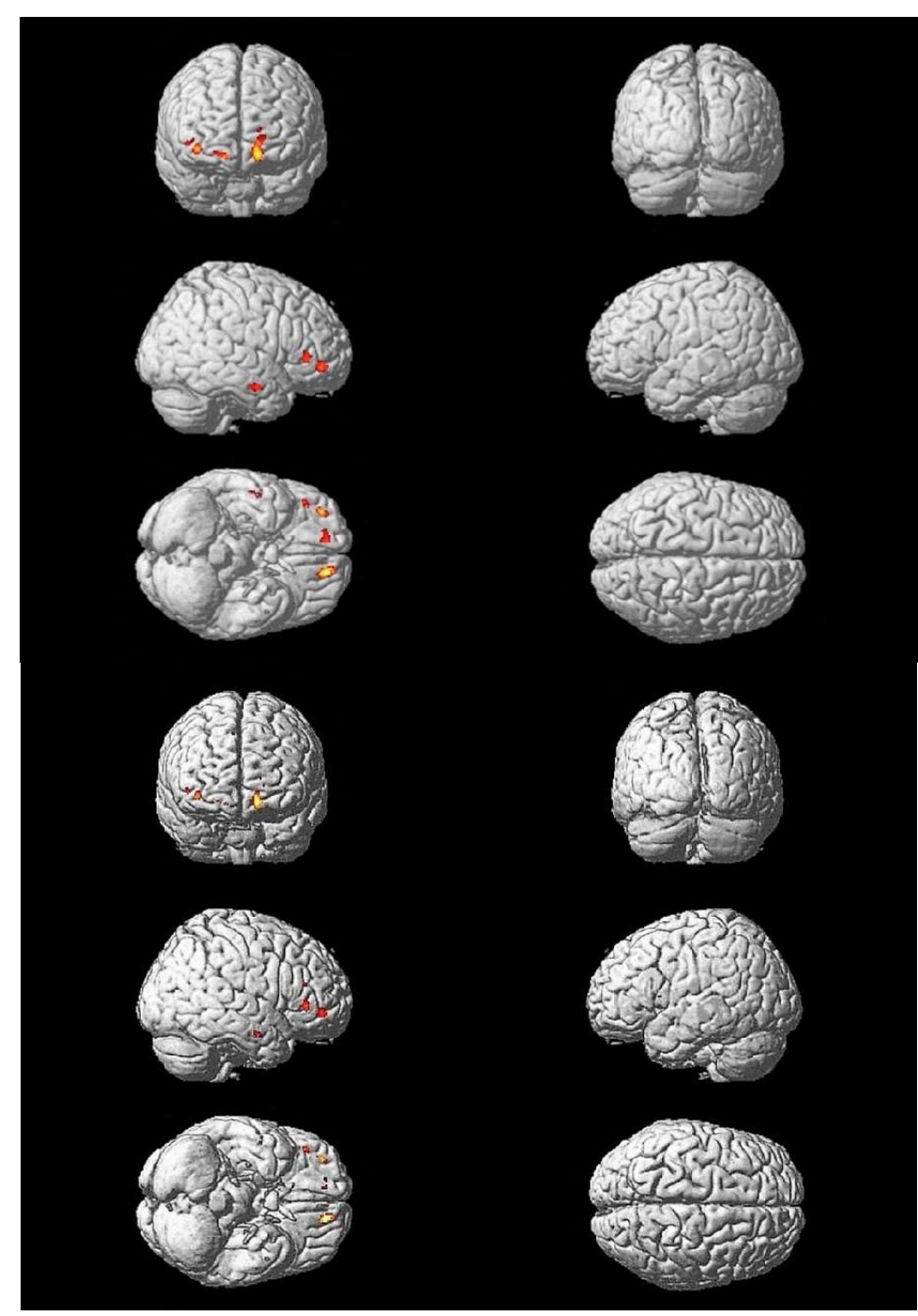

Figure 1. Clusters significantly reduced in all patients compared to controls (up) and only in MDD patients (down)

In addition, a one-way ANOVA model differentiated between the two patient groups (unipolar and bipolar) and the control subjects. Significant difference was found between healthy controls and subjects with major depression again in clusters located to the left MFC (158 voxels) and left ACC (120 voxels), and right middle and inferior frontal gyri (49 and 48 voxels respectively) while the contrast between controls and bipolar patients did not reach statistical significance. See Table 3 for more details. The contrast between the two patients' groups did not survive FDR correction.

Table 3. Clusters of grey matter volumes significantly reduced in MDD patients compared to healthy controls

\begin{tabular}{|c|c|c|c|c|c|}
\hline \multirow[t]{2}{*}{ Anatomical localization } & \multirow{2}{*}{$\begin{array}{l}\text { Cluster size } \\
\text { (voxels) }\end{array}$} & \multicolumn{3}{|c|}{ Peak MNI coordinates } & \multirow{2}{*}{$\begin{array}{l}\text { p-value } \\
\text { (FDR- } \\
\text { corrected) }\end{array}$} \\
\hline & & $\mathrm{x}$ & $\mathrm{y}$ & $\mathrm{z}$ & \\
\hline Left medial frontal cortex & 158 & -12 & 51 & -9 & 0.025 \\
\hline Left anterior cingulate cortex & 120 & -14 & 44 & 11 & 0.025 \\
\hline
\end{tabular}




\begin{tabular}{llllll}
\hline Right middle frontal gyrus & 49 & 38 & 47 & -5 & 0.025 \\
$\begin{array}{l}\text { Right inferior frontal gyrus } \\
\text { (triangular and orbital parts) }\end{array}$ & 48 & 45 & 35 & 0 & 0.025 \\
Right middle temporal gyrus & 22 & 51 & -6 & -23 & 0.030 \\
\hline
\end{tabular}

\section{Discussion}

The results of the present study suggest that patients with mood disorders (unipolar and bipolar depression combined) demonstrate significant decreases in grey matter volume restricted to regions located in medial frontal and anterior cingulate cortex on the left side and middle frontal gyrus, medial orbital gyrus, inferior frontal gyrus (triangular and orbital parts), and middle temporal gyrus (extending to the superior temporal gyrus) on the right side. Nevertheless, when the patient group was divided into bipolar and unipolar depression the reductions remained significant only for the unipolar depression patients. These findings replicate some previous morphometric, original and meta-analytic studies on depression and their significance will be discussed in the following lines.

The biggest cluster showing reduction of grey matter in the patient group in our study was situated in MFC and ACC. Accordingly, in medication naïve MDD patients the GM volume of the MFC was found to be significantly reduced ${ }^{10}$. Similar findings with regard to ACC, were reported by a number of individual studies ${ }^{23-26}$ although there were some negative findings published as well 12,13 . Moreover, a recent meta-analysis on 20 VBM studies confirmed the reduction of the GM in bilateral $\mathrm{ACC}^{6}$. Left and less robust right volumetric reductions in patients with mood disorders, predominantly in those with unipolar depression have been previously identified by Hajek et al. ${ }^{27}$. A much more extensive meta-analysis based on 101 publications of structural MRI outlined the right ACC as significantly smaller in unipolar depression ${ }^{5}$. Interestingly, their meta-regression analysis demonstrated that in the left ACC a larger effect size was associated with increasing severity of illness and later age of onset which might explain the observed discrepancy in the literature on the laterality of the reduction in anterior cingulate GM volume.

In bipolar depression the most prominent structural abnormality as identified by VBM is suggested to be the reduction of the left ACC according to the meta-analytical study of Bora et al. which included 660 patients and 770 healthy control subjects ${ }^{7}$. We might assume that in our study several factors including the heterogeneity of the patient sample (both unipolar and bipolar), and the severity of illness (moderate to severe depression) have contributed to the finding of significant reduction only in the left cingulate region.

The role of the anterior cingulate cortex in depression has been implicated not only by structural but also by functional studies demonstrating changes in task-related and restingstate activity 28,29 . This brain area is involved in cognitive and affective regulation, attention, problem solving, motivation, and decision making, and there is a general agreement on the existence of an affective and a cognitive subdivision ${ }^{30}$. Both have been considered to be involved in the pathophysiology of depression as the disorder manifests with affective and cognitive symptoms alongside ${ }^{31,32}$.

The other significant clusters demonstrating reduction in GM volume in our patient group were located in the orbitofrontal cortex (OFC). This finding is in accordance with at least two meta-analyses 4,5 identifying the $\mathrm{OFC}$ as significantly reduced in depressed patients. Moreover, Arnone et al. found that the effect size of the right OFC was larger with increasing proportion of patients medicated with antidepressants and antipsychotics while duration of illness was associated with smaller effect size ${ }^{11}$. In addition, metabolic and functional studies have consistently provided evidence for disturbances in this area as characteristic for several psychiatric disorders and depression in particular ${ }^{33,34}$. 
As part of the prefrontal cortex, the OFC is involved in the processing of rewards and punishments which is essential for the complex emotional and social human behaviour ${ }^{35}$. A meta-analysis of functional MRI in depression has revealed increased activity of the OFC to positive emotional stimuli ${ }^{36}$. Moreover, treatment response prediction studies suggest that this activity is normalized by antidepressants ${ }^{37}$. During reward processing this same area was found to be hypoactive in unmedicated currently depressed and in remitted patients as well as in young individuals with familial risk for depression ${ }^{38-40}$.

The reduction of the GM volume of a cluster in the superior/middle temporal gyrus in our study is in accordance with the recent meta-analysis of Arnone et al. ${ }^{11}$ where patients with major depression demonstrated significant grey matter loss in the temporal gyri bilaterally. Interestingly, significantly decreased GM volume in the right middle temporal gyrus was found in patients with suicide history compared to healthy controls ${ }^{41}$. Similarly, in bipolar disorder big cluster of GM reduction encompassing the insula, middle temporal gyrus, superior temporal gyrus, temporopolar area, pars opercularis and pars triangularis, inferior frontal gyrus, and claustrum on the right side was identified by a recent meta-analysis ${ }^{42}$.

Dividing the patient group into bipolar and unipolar depression in our study led to negative result for the bipolar patients compared to both MDD patients and healthy controls. This is in accordance with some earlier individual and meta-analytical studies that failed to find significant difference in GM volumes between bipolar patients and healthy subjects 43,44 . These findings contrast our a priori hypothesis that the GM volumes would be similarly affected in both major depressive and bipolar affective disorder. Thus, it might be beneficial to analyse these two patient groups separately but also with a sufficient sample size which is a limitation in our study since the bipolar group consists of only 11 subjects. Yet the effect of the latent, or sub-threshold bipolarity as well as the impact of the bipolar spectrum ${ }^{15}$ should be properly considered as well.

There are not many studies in the available literature that directly compare GM volumes in unipolar and bipolar depression. The only such VBM study by Cai et al. ${ }^{45}$ detected a cluster of reduced GM volume in the middle cingulate gyrus of bipolar I patients. The fact that we were not able to detect any difference between the two patient groups in our study might be due to several reasons including the small sample, the diagnostic heterogeneity (bipolar I and II), the medication status (most of the patients were medicated) or the statistical threshold (the cited article used an uncorrected $\mathrm{p}<0.001$ ).

\section{Conclusion}

In summary, using VBM the present study was able to replicate decreases in GM volume in frontal and temporal regions in patients with major depression and bipolar disorder compared to healthy controls. As shown by the further analysis comparing the two patient groups, the abovementioned results were mainly driven by the difference between the patients with major depression and the healthy individuals. Future studies should examine VBM in patients with major depression and bipolar disorder separately as well as in patients with BD1 and BD2. This may improve the characterization of the structural abnormalities in mood disorders and their subtypes. Moreover, the bias of the sub-syndromal and latent forms of bipolarity should be taken into consideration and further investigations should be performed in order to produce accurate biological measures differentiating the two clinical diagnoses. In this respect, it is interesting to note that there are highly significant differences in neuro-oxidative pathways between unipolar depression and bipolar depression with increased damage to lipids and proteins coupled with aldehyde formation in unipolar depression as compared with $\mathrm{BD} 1$ and BD2 ${ }^{46}$. The greater neuro-oxidative damage observed in unipolar depression may explain the greater neurodegenerative or neuroprogressive aberrations in grey matter volume as detected in the present study ${ }^{46,47}$. 


\section{Acknowledgements}

Imaging data were acquired at the Research Complex for Translational Neuroscience, Medical University of Plovdiv and the authors would like to thank the radiographer Donka Popova for her devotion to this project. The patient recruitment was assisted by Zlatoslav Arabadzhiev, MD, PhD and valuable support has been offered by Stefan Kostyanev, MD, $\mathrm{PhD}$, we are grateful to both.

\section{References}

1. Sartorius N. The economic and social burden of depression. J Clin Psychiatry. 2001;62 Suppl 15:8-11.

2. Drevets WC, Price JL, Furey ML. Brain structural and functional abnormalities in mood disorders: implications for neurocircuitry models of depression. Brain Struct Funct. 2008;213(1-2):93-118.

3. Sacher J, Neumann J, Funfstuck T, Soliman A, Villringer A, Schroeter ML. Mapping the depressed brain: a meta-analysis of structural and functional alterations in major depressive disorder. J Affect Disord. 2012;140(2):142-148.

4. Koolschijn PC, van Haren NE, Lensvelt-Mulders GJ, Hulshoff Pol HE, Kahn RS. Brain volume abnormalities in major depressive disorder: a meta-analysis of magnetic resonance imaging studies. Hum Brain Mapp. 2009;30(11):3719-3735.

5. Arnone D, McIntosh AM, Ebmeier KP, Munafo MR, Anderson IM. Magnetic resonance imaging studies in unipolar depression: systematic review and meta-regression analyses. Eur Neuropsychopharmacol. 2012;22(1):1-16.

6. Lai CH. Gray matter volume in major depressive disorder: a meta-analysis of voxel-based morphometry studies. Psychiatry Res. 2013;211(1):37-46.

7. Bora E, Fornito A, Yucel M, Pantelis C. Voxelwise meta-analysis of gray matter abnormalities in bipolar disorder. Biol Psychiatry. 2010;67(11):1097-1105.

8. Kempton MJ, Salvador Z, Munafo MR, et al. Structural neuroimaging studies in major depressive disorder. Meta-analysis and comparison with bipolar disorder. Arch Gen Psychiatry. 2011;68(7):675-690.

9. Zhao YJ, Du MY, Huang XQ, et al. Brain grey matter abnormalities in medication-free patients with major depressive disorder: a meta-analysis. Psychol Med. 2014;44(14):2927-2937.

10. Lai $\mathrm{CH}, \mathrm{Wu}$ YT. Frontal-insula gray matter deficits in first-episode medication-naive patients with major depressive disorder. J Affect Disord. 2014;160:74-79.

11. Arnone D, Job D, Selvaraj $S$, et al. Computational meta-analysis of statistical parametric maps in major depression. Hum Brain Mapp. 2016;37(4):1393-1404.

12. Brambilla P, Nicoletti MA, Harenski K, et al. Anatomical MRI study of subgenual prefrontal cortex in bipolar and unipolar subjects. Neuropsychopharmacology. 2002;27(5):792-799.

13. Bremner JD, Vythilingam $M$, Vermetten $E$, et al. Reduced volume of orbitofrontal cortex in major depression. Biol Psychiatry. 2002;51(4):273-279.

14. Schmaal L, Hibar DP, Samann PG, et al. Cortical abnormalities in adults and adolescents with major depression based on brain scans from 20 cohorts worldwide in the ENIGMA Major Depressive Disorder Working Group. Mol Psychiatry. 2017;22(6):900-909.

15. Angst J, Gamma A. A new bipolar spectrum concept: a brief review. Bipolar Disord. 2002;4 Suppl 1:11-14.

16. Ghaemi SN. Bipolar Spectrum: A Review of the Concept and a Vision for the Future. Psychiatry Investig. 2013;10(3):218-224.

17. Haralanov S, Haralanova E, Terziivanova P, Moeller H-J, Hennig-Fast K. Subjective Emotional Over-arousal to Neutral Social Scenes in Paranoid Schizophrenia Psychosis Is Significantly Reduced by Effective Antipsychotic Pharmacotherapy. American Journal of Psychology and Cognitive Science. 2015;1(2):48-60. 
18. Perlis $\mathrm{RH}$, Uher $\mathrm{R}$, Ostacher $\mathrm{M}$, et al. Association between bipolar spectrum features and treatment outcomes in outpatients with major depressive disorder. Arch Gen Psychiatry. 2011;68(4):351-360.

19. Mazza M, Mandelli L, Zaninotto L, et al. Factors associated with the course of symptoms in bipolar disorder during a 1-year follow-up: depression vs. sub-threshold mixed state. Nord J Psychiatry. 2011;65(6):419-426.

20. Haralanov S, Terziivanova P. Subclinical bipolarity in unipolar depression: objective revealing by computerized ultrasonographic cranio-corpo-graphy. Bulgarian Medicine. 2011;1(3):12.

21. Sheehan DV, Lecrubier Y, Sheehan KH, et al. The Mini-International Neuropsychiatric Interview (M.I.N.I.): the development and validation of a structured diagnostic psychiatric interview for DSM-IV and ICD-10. J Clin Psychiatry. 1998;59 Suppl 20:22-33;quiz 34-57.

22. Montgomery SA, Asberg M. A new depression scale designed to be sensitive to change. $\mathrm{Br} J$ Psychiatry. 1979;134:382-389.

23. Ballmaier M, Toga AW, Blanton RE, et al. Anterior cingulate, gyrus rectus, and orbitofrontal abnormalities in elderly depressed patients: an MRI-based parcellation of the prefrontal cortex. Am J Psychiatry. 2004;161(1):99-108.

24. Coryell W, Nopoulos P, Drevets W, Wilson T, Andreasen NC. Subgenual prefrontal cortex volumes in major depressive disorder and schizophrenia: diagnostic specificity and prognostic implications. Am J Psychiatry. 2005;162(9):1706-1712.

25. Tang Y, Wang F, Xie G, et al. Reduced ventral anterior cingulate and amygdala volumes in medication-naive females with major depressive disorder: A voxel-based morphometric magnetic resonance imaging study. Psychiatry Res. 2007;156(1):83-86.

26. Caetano SC, Kaur S, Brambilla P, et al. Smaller cingulate volumes in unipolar depressed patients. Biol Psychiatry. 2006;59(8):702-706.

27. Hajek T, Kozeny J, Kopecek M, Alda M, Hoschl C. Reduced subgenual cingulate volumes in mood disorders: a meta-analysis. J Psychiatry Neurosci. 2008;33(2):91-99.

28. Salvadore G, Cornwell BR, Colon-Rosario V, et al. Increased anterior cingulate cortical activity in response to fearful faces: a neurophysiological biomarker that predicts rapid antidepressant response to ketamine. Biol Psychiatry. 2009;65(4):289-295.

29. Davey CG, Harrison BJ, Yucel M, Allen NB. Regionally specific alterations in functional connectivity of the anterior cingulate cortex in major depressive disorder. Psychol Med. 2012;42(10):2071-2081.

30. Bush G, Luu P, Posner MI. Cognitive and emotional influences in anterior cingulate cortex. Trends Cogn Sci. 2000;4(6):215-222.

31. Jaworska N, Yang XR, Knott V, MacQueen G. A review of fMRI studies during visual emotive processing in major depressive disorder. World J Biol Psychiatry. 2015;16(7):448-471.

32. Wang YL, Yang SZ, Sun WL, Shi YZ, Duan HF. Altered functional interaction hub between affective network and cognitive control network in patients with major depressive disorder. Behav Brain Res. 2016;298(Pt B):301-309.

33. Drevets WC. Orbitofrontal cortex function and structure in depression. Ann N Y Acad Sci. 2007;1121:499-527.

34. Jackowski AP, Araujo Filho GM, Almeida AG, et al. The involvement of the orbitofrontal cortex in psychiatric disorders: an update of neuroimaging findings. Rev Bras Psiquiatr. 2012;34(2):207-212.

35. Kringelbach ML, Rolls ET. The functional neuroanatomy of the human orbitofrontal cortex: evidence from neuroimaging and neuropsychology. Prog Neurobiol. 2004;72(5):341-372.

36. Groenewold NA, Opmeer EM, de Jonge P, Aleman A, Costafreda SG. Emotional valence modulates brain functional abnormalities in depression: evidence from a meta-analysis of fMRI studies. Neurosci Biobehav Rev. 2013;37(2):152-163. 
37. Delaveau P, Jabourian M, Lemogne C, Guionnet S, Bergouignan L, Fossati P. Brain effects of antidepressants in major depression: a meta-analysis of emotional processing studies. $J$ Affect Disord. 2011;130(1-2):66-74.

38. McCabe C, Cowen PJ, Harmer CJ. Neural representation of reward in recovered depressed patients. Psychopharmacology (Berl). 2009;205(4):667-677.

39. McCabe C, Woffindale C, Harmer CJ, Cowen PJ. Neural processing of reward and punishment in young people at increased familial risk of depression. Biol Psychiatry. 2012;72(7):588-594.

40. Ubl B, Kuehner C, Kirsch P, Ruttorf M, Diener C, Flor H. Altered neural reward and loss processing and prediction error signalling in depression. Soc Cogn Affect Neurosci. 2015;10(8):1102-1112.

41. Peng $\mathrm{H}, \mathrm{Wu} \mathrm{K}, \mathrm{Li} \mathrm{J}$, et al. Increased suicide attempts in young depressed patients with abnormal temporal-parietal-limbic gray matter volume. J Affect Disord. 2014;165:69-73.

42. Selvaraj S, Arnone D, Job D, et al. Grey matter differences in bipolar disorder: a metaanalysis of voxel-based morphometry studies. Bipolar Disord. 2012;14(2):135-145.

43. McDonald C, Zanelli J, Rabe-Hesketh S, et al. Meta-analysis of magnetic resonance imaging brain morphometry studies in bipolar disorder. Biol Psychiatry. 2004;56(6):411-417.

44. Scherk H, Kemmer C, Usher J, Reith W, Falkai P, Gruber O. No change to grey and white matter volumes in bipolar I disorder patients. Eur Arch Psychiatry Clin Neurosci. 2008;258(6):345-349.

45. Cai Y, Liu J, Zhang L, et al. Grey matter volume abnormalities in patients with bipolar I depressive disorder and unipolar depressive disorder: a voxel-based morphometry study. Neurosci Bull. 2015;31(1):4-12.

46. Maes M, Landucci Bonifacio K, Morelli NR, et al. Major Differences in Neurooxidative and Neuronitrosative Stress Pathways Between Major Depressive Disorder and Types I and II Bipolar Disorder. Mol Neurobiol. 2019;56(1):141-156.

47. Maes M, Yirmyia R, Noraberg J, et al. The inflammatory \& neurodegenerative (I\&ND) hypothesis of depression: leads for future research and new drug developments in depression. Metab Brain Dis. 2009;24(1):27-53. 